\title{
Recognition of practical English speech emotion using improved Quantum Ant Colony Algorithm
}

\author{
Lihui DU ${ }^{1, a}$ Yueguang $\mathrm{Li}^{2, \mathrm{~b}}$ \\ ${ }^{1}$ Gansu Normal University for Nationalities, Hezuo, gansu, 747000, \\ China \\ ${ }^{2}$ Gansu Normal University for Nationalities, Hezuo, gansu, 747000, \\ China

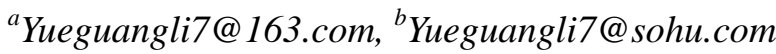

\begin{abstract}
Due to the drawbacks in Support Vector Machine (SVM) parameter optimization, an improved quantum ant colony algorithm was proposed, and the learning ability in practical English speech emotion recognition was improved. The experimental results showed that quantum ant colony algorithm may significantly improve the practical English speech emotion recognition.
\end{abstract}

Keywords: improved Quantum Ant Colony Algorithm; English speech emotion; Recognition; heuristics

\section{Introduction}

Recently as the fast development of computer technology, natural human-computer interaction is becoming more and more important. English speech emotion recognition is one of the key technologies in natural human-computer interaction, which has drawn more and more attention from researchers from psychology, computer science and cognitive science. As a new research topic, research efforts are focused on basic speech emotions including happiness, sadness, anger and neutrality ${ }^{[1]}$. However, there are various practical emotions in the real world application, such as fidgetiness, and. the limited basic 
English speech emotion recognition research may not satisfy the demands in the real world ${ }^{[2]}$.

The most common English speech emotion recognition methods include Artificial Neural Network (ANN) ${ }^{[3]}$, Hidden Markov Model (HMM) ${ }^{[4]}$, and Gaussian Mixture Model (GMM) ${ }^{[5]}$. ANN is one of the earliest methods used in speech emotion recognition, Nicholson analyzed the basic emotion types and classified the positive emotions and the negative emotions ${ }^{[6]}$, however the averaged recognition rate was only 50\%. HMM adopts temporal features, which are influenced by text variations, for instance, formant feature is a commonly used speech emotion feature, and it is also strongly influenced by phoneme information. GMM is the state-of-the-art in speaker and language identification, but it is largely dependent on the training data and the mixture number is difficult to set in the experiment, therefore the generalization across various applications and databases needs to be improved.

\section{SVM classification method based on quantum ant colony algorithm}

Considering the annealing mechanism in SA algorithm and the idea of IV, the IV operation is introduced to the global information exchange stage in quantum ant colony algorithm:

(1) Find the current global optimal individual $X_{g}$ as a vaccine;

(2) According to the vaccination probability p, randomly select the information of dimension bits for each individual frog. Replace the corresponding bits of the current individual frog with those of the vaccine and then get the vaccinated individual;

(3) Accept the vaccinated individual according to the annealing mechanism, that is select the frog individual according to the annealing probability: accept the vaccinated individual when $\min \{1, \exp (-\Delta f / T)\}>\operatorname{rand}()$, where $\Delta f$ is the difference of the fitness value before and after vaccination, otherwise keep the original individual, thus completing the IV operation.

This operation improves the quality of candidate solutions greatly, and as the annealing temperature drops, the probability of adopting a bad solution drops, 
therefore the optimization efficiency and accuracy are improved, which makes the global searching ability be strengthened. Meanwhile, the Gaussian mutation and chaotic disturbance operations are introduced in the local depth searching stage of each sub-population. Compare the individual fitness value in the sub-population with the average fitness value of the current sub-population, when the individual fitness value is better, perform Gaussian mutation: $\operatorname{mutation}(x)=x(1+N(0,1))$, where $\mathrm{x}$ stands for the current individual, $N(0,1)$ stands for normal distribution random number with zero mean and one as the standard variance, and then accept the individual after mutation according to annealing probability in the SA algorithm. Otherwise, perform chaotic disturbance, and accept the individual according to the annealing probability. This operation improves the diversity of the population in the later stage of the iteration, the ability of jumping out the local extreme and the convergence speed, and the global optimal value may be achieved finally. In which, the chaotic disturbance process is described below:

(1) Generate a d-dimensional chaotic variable $X=\left(X_{1}, X_{2} \ldots, X_{l} \ldots, X_{d}\right)$ randomly, where $X_{l} \in[0,1], l=1,2, \ldots, d$;

(2) Perform Tent chaotic map on $X^{[7]}$ :

$$
X_{l}^{\prime}=\left\{\begin{array}{lc}
2 X_{l} & 0 \leq X_{l} \leq 1 / 2 \\
2\left(1-X_{l}\right) & 1 / 2 \leq X_{l} \leq 1
\end{array}\right.
$$

(3) Map back $X_{l}^{\prime}$ to the original optimization space:

$$
\text { new } X_{l}=\min _{l}+\left(\max _{l}-\min _{l}\right) X_{l}^{\prime}
$$

where, $\left[\min _{l}, \max _{l}\right]$ is the domain of the $l$-th dimensional variable in the original optimization space. Then the new individual is got after chaotic disturbance: 
new $X=\left(\right.$ new $X_{1}$, new $X_{2} \ldots$, new $X_{1} \ldots$, new $\left.X_{d}\right)$

(4) For the current individual $\mathrm{Y}$ (whose fitness value is worse than the average fitness value of the current sub-population), the new individual after chaotic disturbance is:

new $Y=(Y+$ new $Y) / 2$

\section{Improved quantum ant colony algorithm}

The main idea of the algorithm is: a random moving no load ants encounter an object, and around it, the same objects less, then pick up the objects of higher probability; A random moving load ant, if the surrounding, the same objects more, then it drops the object probability is large. Set a parameter $\beta$, and $\beta$ has a relationship with the population density around the site, $\beta=p * d$. Using many ants, the urban bus station site selection model based on the least running time, forming a plurality of cell, if the value is greater than $\beta$, the site is retained; if the value is less than $\beta$, the site is redundant, it is removed, thereby determining the site address and number. The basic steps of algorithm are as follows:

(1)Initialization: the population of $\mathrm{n}$ individuals $P(t)=\left(p_{1}^{t}, p_{2}^{t} \ldots p_{n}^{t}\right)$, where $p^{t}{ }_{j}\left(j_{=1,2, \ldots,}{ }^{n}\right)$ for the $\mathrm{j}$ individual of the $t$ and its description such as:

$$
p_{j=}^{t}=\left(\begin{array}{l|l|l|l}
\alpha_{1}{ }^{t} & \alpha_{2}{ }^{t} & \ldots & \alpha^{t} \\
\beta_{1}^{t} & \beta_{2}{ }^{t} & \ldots & \beta_{m}{ }^{t}
\end{array}\right)
$$


Where $\mathrm{m}$ is the number of quantum bits, in the beginning, all $\alpha_{i}, \beta_{i}(i=$ $1,2, \ldots, m$ ) are $1 / \sqrt{2}$. The initial number of iterations $t=0$;

(2) According to the probability amplitude values in the case $P(t)$ construct $\mathrm{R}$

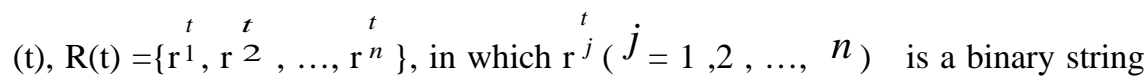
of length $m$, in which each element is determined by the $\left|\alpha_{i}^{t}\right|^{2}$ and $\left|\beta_{i}^{t}\right|^{2}$ $(i=1,2, \ldots, m)$ in $\left.p^{t}{ }_{j=1,2, \ldots,} n\right)$. Set a random number $\mathrm{w}(\mathrm{w} \in[0,1])$, if $\left|\alpha_{i}^{t}\right|^{2}>\mathrm{w}$, then $\mathrm{r}^{\boldsymbol{t}}=0$, or r ${ }^{\boldsymbol{t}}=1(i=1,2, \ldots m)$;

(3) The total number of candidate sites is $\mathrm{k}$. $n$ ants will be placed in one of the $\mathrm{k}$ points randomly;

(4)Define a tabu search table $\mathrm{vb}$ and its initial value is 0 ,if $\mathrm{vb}\left(k_{i}\right)=1$, it expresses the ant chose the site $i(i=1,2, \cdots, \mathrm{k})$, if $\mathrm{vb}\left(k_{i}\right)=0$, it expresses the ant gave up the site $i \quad(i=1,2, \cdots, k)$;

(5) Ants through repeated application of state transition rules, according to Equation (7) to established a path, and in the process of establishing the path of each step. Its description is in equation (6). Until all ants have completed the construction of the solution path;

$\beta_{i}(a, b)=\left\{\begin{array}{lc}\frac{d_{(a, b)}-E_{a}}{L_{a}} * 0.5+0.5 & (a \neq b) \wedge\left(L_{a} \neq 0\right) \\ 0 & (a=b \\ 0.5 & (a \neq b) \wedge\left(L_{a}=0\right)\end{array}\right.$

(6) Record the optimal solution ;

(7) Update the amount of pheromone of the path to use the rules of quantum rotation gate; 
(8) If meet the output conditions (such as the maximum evolution generation $\mathrm{t}^{\max }=2000$ ), then the optimal solution has been reached, otherwise set $\mathrm{t}=\mathrm{t}+1$ and return to(3).

\section{The analysis of simulation}

In order to investigate the feasibility and effectiveness of the improved quantum ant colony algorithm, improved quantum ant colony algorithm was tested on the practical English speech emotion database. The experimental results showed that first type error ratio is the sum rate of the misclassification of an emotion as other emotions, and the second type error ratio is the sum rate of the misclassification of other emotions as the target emotion.

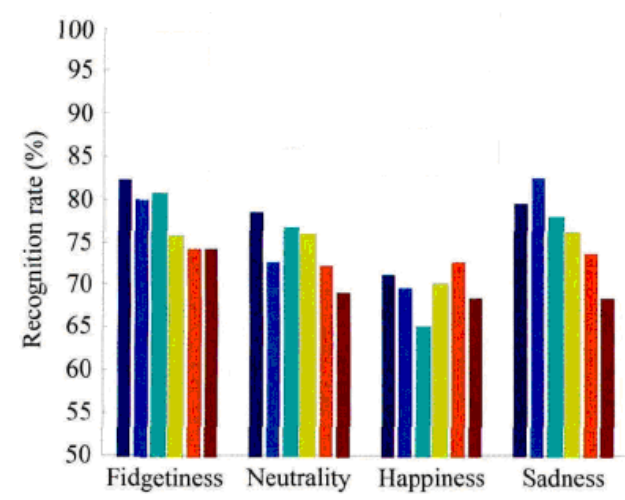

(a) Comparison of recognition rates

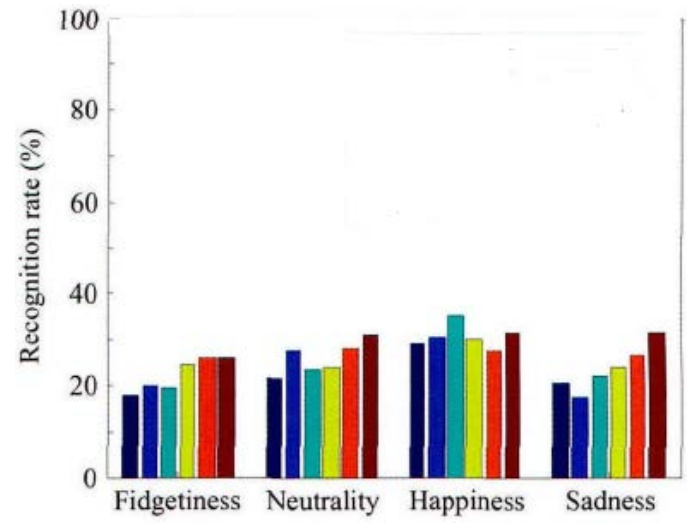

(b) Comparison of first type error ratios 


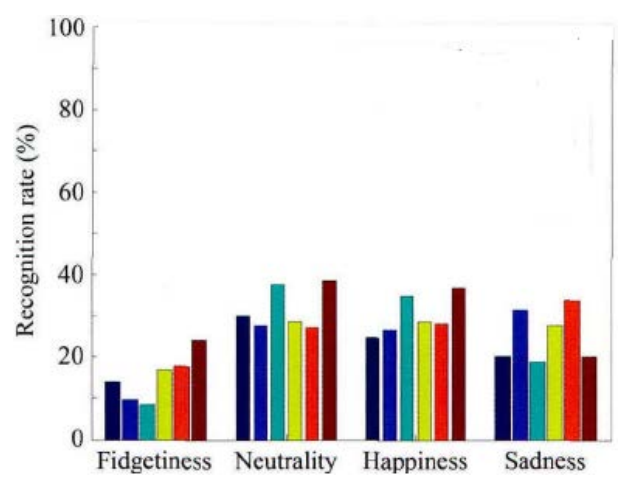

(c) Comparison of second type error ratios

Fig.1 Comparison of ratios

\section{Conclusions}

This paper proposes an improved quantum ant colony algorithm, the improved quantum ant colony algorithm is applied to solve practical English speech emotion, the performance has been improved obviously. Experiments show that: The algorithm is effective and robust, it is feasible and effective for solving the practical English speech emotion.

\section{References}

[1] Pao T L, Chen Y, Yeh J H. Emotion recognition and evaluation from mandarin speech signals. International Journal of Innovative Computing, Information and Control, 2008, 4(7): 1695-1709.

[2] Zhao Y, Zhao L, Zou C R al. Speech emotion recognition using modified quadratic discrimination function. Journal of Electronics (China), 2008, 25(6): 840-844

[3] Dai K S, Fell H J, MacAuslan J. Recognizing emotion in speech using neural networks. In: Proc. of the IASTED International Conference on Telehealth/Assistive Technologies. Baltimore, USA, 2008: 31-36.

[4] Nwe T L, Foo S W, Silva LCD. Speech emotion recognition using hidden Markov models. Speech Communication, 2003, 41(4): 603-623.

[5] Huang C W, Zhao Y, Jin Y et, al A Study on Feature Analysis and Recognition of Practical Speech Emotion. Journal of Electronics \& Information Technology, 2011; 33(1): 112-116. 
[6] Nicholson J, Takahashi K, Nakatsu R. Emotion recognition in speech using neural networks. Neural Computing k. Applications, 2000, 9: 290-296.

[7] Wang X Y, Wang L L. A new perturbation method to the Tent map and its application. Chinese Physics B, 2011, 20(5): 191-198. 